\title{
Nanoscale magnetic configurations of supported Fe nanoparticle assemblies studied by scanning electron microscopy with spin analysis
}

\author{
Wen-Chin Lin* \\ Department of Physics, National Taiwan Normal University, 11677 Taipei, Taiwan \\ and Department of Physics, National Taiwan University, 10617 Taipei, Taiwan \\ Zheng Gai, Lan Gao, and Jian Shen \\ Center for Nanophase Materials Science Division, Oak Ridge National Laboratory, Oak Ridge, Tennessee 37831, USA \\ and Materials Science and Technology Division, Oak Ridge National Laboratory, Oak Ridge, Tennessee 37831, USA \\ Pin-Jui Hsu and Hong-Yu Yen \\ Department of Physics, National Taiwan University, 10617 Taipei, Taiwan \\ Minn-Tsong $\operatorname{Lin}^{\dagger}$ \\ Department of Physics, National Taiwan University, 10617 Taipei, Taiwan \\ and Institute of Atomic and Molecular Sciences, Academia Sinica, 10617 Taipei, Taiwan \\ (Received 13 April 2009; revised manuscript received 17 June 2009; published 8 July 2009)
}

\begin{abstract}
Microscopic magnetic behavior of supported nanoparticles is strongly correlated with their functionalities, especially in data storage and biological applications, but still needs to be clarified. We studied nanoscale magnetic configurations of Fe nanoparticle assemblies using scanning electron microscopy with polarization analysis. The flux closure domain configurations and the reduced magnetic correlation length $(\sim 250 \mathrm{~nm})$, relative to the conventional thin films, are determined. Quantitative analysis indicates the magnetic interaction energy to be 80-99 meV, close to the magnetic dipolar coupling energy. These direct observations evidence the aforereported simulations and will be valuable for fabricating magnetic nanoparticle assemblies with the desired magnetic properties.
\end{abstract}

DOI: $10.1103 /$ PhysRevB.80.024407

PACS number(s): 75.60.Ch, 75.75.+a, 75.25.+z

\section{INTRODUCTION}

Various kinds of magnetic nanoparticles are studied in the last decade, in order to control and design the magnetic properties for applications of data storage, magnetoelectronic device, and drug delivery. ${ }^{1-6}$ For each of the purposes, it is unavoidable to have the nanoparticles adsorbing on micron or nanoscale supporting surface. The functionalities of the nanoparticles are strongly correlated with their local collective magnetic behaviors. ${ }^{3-6}$ For example, the magnetic domain size should directly correspond to the possible area size of active function. Therefore the microscopic magnetic collective behavior of nanoparticles, such as magnetic domain configuration and correlation length, is crucial for utilization in these applications. Due to the limitation of experimental tools, detailed studies based on microscopic imaging, however, are still lacking.

Basically, magnetic behavior is determined by the intrinsic exchange coupling energy and anisotropy energy of the materials. Decreasing the single particle size usually reduces the anisotropy energy or modifies the easy direction. If considering the magnetic nanoparticle assembly, the particleparticle interaction becomes an important issue, dominating the collective magnetic behavior. ${ }^{7}$ Through changing the particle density, interdistance, or space arrangement, the magnetic properties might be controlled by design. ${ }^{8,9}$ From theoretical point of view, several models have been proposed to consider the influence of dipolar interaction, multiple interaction, tunneling exchange coupling, etc. ${ }^{7,10-12}$ On the ex- perimental front, most measurements have been carried out using averaging techniques such as magneto-optical Kerr effect (MOKE) and superconducting quantum interference device (SQUID). Studies based on microscopic imaging, however, are usually difficult to carry out. Scanning probe techniques such as spin-polarized scanning tunneling microscopy (SP-STM) (Ref. 13) and magnetic force microscopy (MFM), ${ }^{14-17}$ while being powerful for magnetic domain imaging, are generally difficult to be used for imaging nanoparticle assemblies due to the significant interference of the morphological corrugation. In contrast, scanning electron microscopy with polarization analysis (SEMPA) is little affected by morphology and gives a vector signal that is directly proportional to magnetization. The latter is in stark contrast to SP-STM and MFM which only measure nonvector signals that are indirectly related to magnetization. In this letter, an ultrahigh vacuum (UHV) SEMPA is utilized to study the microscopic magnetic behavior of nanoparticle assemblies. ${ }^{18}$ Detailed domain size and magnetization configuration are observed clearly. STM is employed to characterize the particle size and spatial distribution. The combination of in situ SEMPA and STM brings the high-resolution magnetic and topographic imaging together on the ideal model system of supported nanoparticle assemblies, without mutual interference and contamination effect. A simple method is also proposed to estimate the magnetic interaction between particles, based on the SEMPA measurements. 
(a) 9 ML Fe

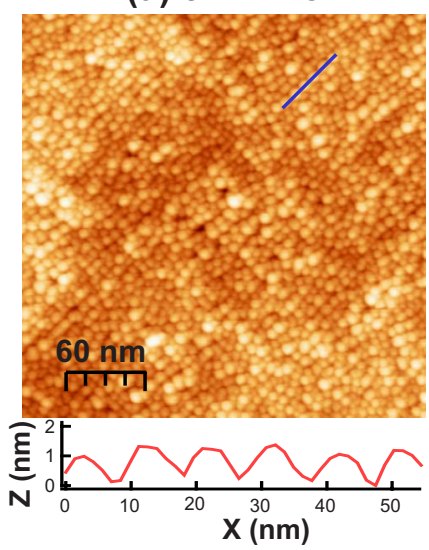

(b) 13 ML Fe

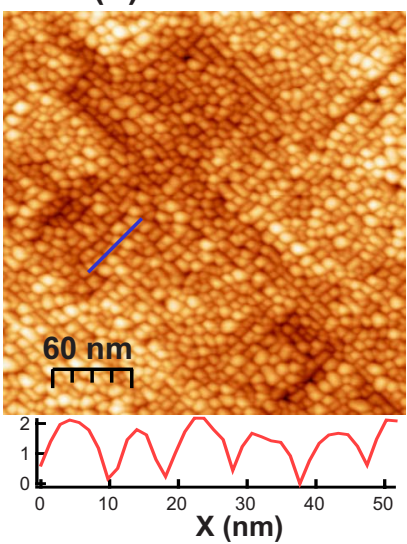

FIG. 1. (Color online) STM images of (a) 9 and (b) 13 ML Fe nanoparticle assemblies on $\mathrm{Al}_{2} \mathrm{O}_{3} / \mathrm{NiAl}(100)$ with the indicated line profiles shown in the bottom. (Image size: $300 \times 300 \mathrm{~nm}$ )

\section{EXPERIMENT}

The experiments were performed in UHV chambers with base pressure better than $2 \times 10^{-10}$ mbar. All the sample preparation and characterization are in situ carried out. After cycles of $\mathrm{Ar}^{+}$sputtering and annealing, the $\mathrm{Al}_{2} \mathrm{O}_{3} / \mathrm{NiAl}(100)$ template was prepared by oxidation of a $\mathrm{NiAl}(100)$ single crystal at $1000 \mathrm{~K} .^{2,3,19} \mathrm{Fe}$ nanoparticles were grown by direct deposition of $\mathrm{Fe}$ atoms onto the $\mathrm{Al}_{2} \mathrm{O}_{3} / \mathrm{NiAl}(100)$ template at room temperature (RT). Note that the growth of $\mathrm{Fe}$ on $\mathrm{Al}_{2} \mathrm{O}_{3} / \mathrm{NiAl}(100)$ forms particles, while $\mathrm{Fe}$ grown on $\mathrm{NiAl}(100)$ forms films. The nominal thickness of $\mathrm{Fe}$ nanoparticles is expressed in a unit of monolayer (ML), which is defined as the atom density on $\mathrm{Cu}(100)$ surface: 1.54 $\times 10^{15}$ at. $/ \mathrm{cm}^{2}$, since the deposition rate was calibrated from the epitaxial growth on $\mathrm{Cu}(100)$. The morphology and growth of $\mathrm{Fe}$ nanoparticles have been reported in our previous work. ${ }^{20}$ SEMPA was used to characterize the magnetic domain configuration of the as grown Fe nanoparticle assemblies at RT, without any magnetic treatment. The macroscopic magnetic behavior was investigated by MOKE. The sample qualities, such as the size distribution etc., were confirmed by STM.

\section{RESULTS AND DISCUSSION}

Figure 1 shows the STM images of Fe nanoparticle assemblies on $\mathrm{Al}_{2} \mathrm{O}_{3} / \mathrm{NiAl}(100)$ with the deposition coverage $=9$ and 13 ML. The particle size increases with deposition coverage. The gaps between nanoparticles are observable, and the shape of nanoparticles also sustains. Although the particles are grown on an insulating layer, we cannot exclude the possible connection at particle base. The full width at half maximum (FWHM) of size distribution does not increase significantly with coverage. Particles of very large size $(>10 \mathrm{~nm})$ are seldom seen. The significant reduction in Curie temperature of nanoparticle assemblies, as compared with the thin films, is consistent with the fact that the nanoparticles are still separated, at least in the sense of long-range exchange coupling. ${ }^{21}$ The future performance of the transport (a) $9 \mathrm{ML} \mathrm{Fe} / \mathrm{Al}_{2} \mathrm{O}_{3} / \mathrm{NiAl}(100)$

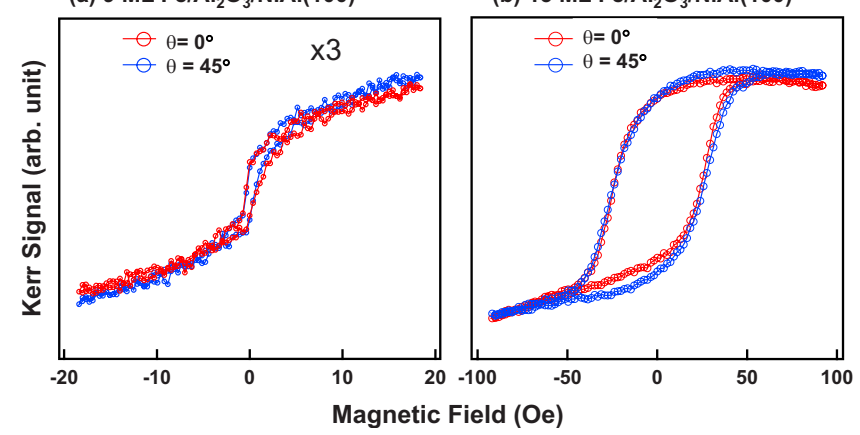

FIG. 2. (Color online) In-plane MOKE hysteresis loops of (a) 9 and (b) $13 \mathrm{ML} \mathrm{Fe}$ nanoparticle assemblies on $\mathrm{Al}_{2} \mathrm{O}_{3} / \mathrm{NiAl}(100)$. $\theta=0^{\circ}$ and $45^{\circ}$ are measured with the magnetic field along [010] and [011] directions, respectively.

measurement would be interesting for clarifying the connection between nanoparticles.

$\mathrm{Fe}$ nanoparticle assemblies prepared in this experiment reveal in-plane magnetic anisotropy. ${ }^{21}$ Fig. 2 exhibits the inplane MOKE hysteresis loops of 9 and 13 ML Fe nanoparticle assemblies recorded at $300 \mathrm{~K}$ (RT). The hysteresis loops measured with magnetic field along [010] and [011] look similar, indicating no preferred easy axis in the plane. This is reasonable since the shape of nanoparticles is isotropic in the plane and the particle alignment seems insignificant.

One can imagine that during the evaporation of $\mathrm{Fe}$, the particle size was getting larger and the particles were becoming closer to each other. Meanwhile the magnetic particleparticle interaction was getting stronger and gradually formed the magnetic domain. Thus the as grown state, without applying any magnetic field, is chosen for the SEMPA measurement and for the estimation of correlation length. Figure 3 shows the SEMPA images of (a) 9 ML Fe nanoparticles, (b) 13 ML Fe nanoparticles and (c) 9 ML Fe film with the histogram of the magnetization angle. The arrows indicate the magnetization directions. The SEMPA images shown in Figs. 3(a) and 3(b) were taken sequentially at the same scanning area, with increasing $\mathrm{Fe}$ coverage. Thus the images look similar. However, we can still observed that some small domains and some frustrated configurations disappeared when increasing $\mathrm{Fe}$ coverage from 9 to $13 \mathrm{ML}$.

From the histogram of the magnetization angle, the $9 \mathrm{ML}$ Fe film apparently exhibits anisotropic magnetization with preferred easy axes. In contrast, the preferred magnetization direction is rather unclear in 9 and $13 \mathrm{ML} \mathrm{Fe}$ nanoparticles, which is consistent with the MOKE measurement in Fig. 2. Although the Fe film and the Fe nanoparticles are all inplane magnetized, the domain sizes are extremely different. In Fig. 3(d), the correlation function of magnetization directions of Fe nanoparticle assemblies are summarized, suggesting the magnetic correlation length to be $\sim 250 \mathrm{~nm}$, which is at least 2-3 orders of magnitude smaller than that of the $\mathrm{Fe}$ film (Fig. 3(c): $>200 \mu \mathrm{m}$ ). The large domain size for $\mathrm{Fe}$ film is consistent with the general knowledge that domains of in-plane magnetized ferromagnetic thin films are typically on the scale from $\mu \mathrm{m}$ to $\mathrm{mm}$. The much smaller domain size observed for Fe nanoparticles is likely caused by the reduced magnetic coupling and anisotropy energy in Fe nanoparticle 
(a) $9 \mathrm{ML} \mathrm{Fe} / \mathrm{Al}_{2} \mathrm{O}_{3} / \mathrm{NiAl}(100)$

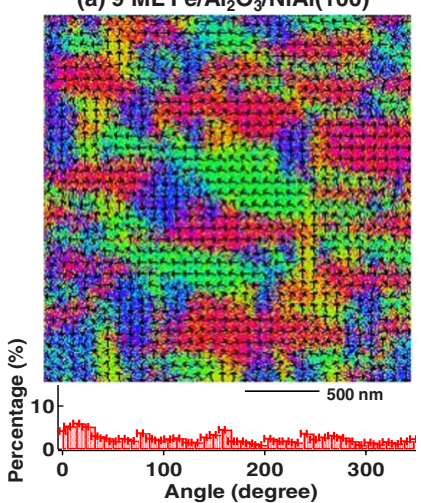

(c) 9 ML Fe/NiAl(100)

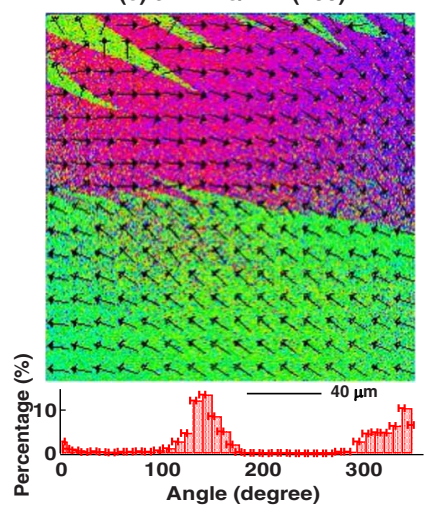

(b) $13 \mathrm{ML} \mathrm{Fe} / \mathrm{Al}_{2} \mathrm{O}_{3} / \mathrm{NiAl}(100)$
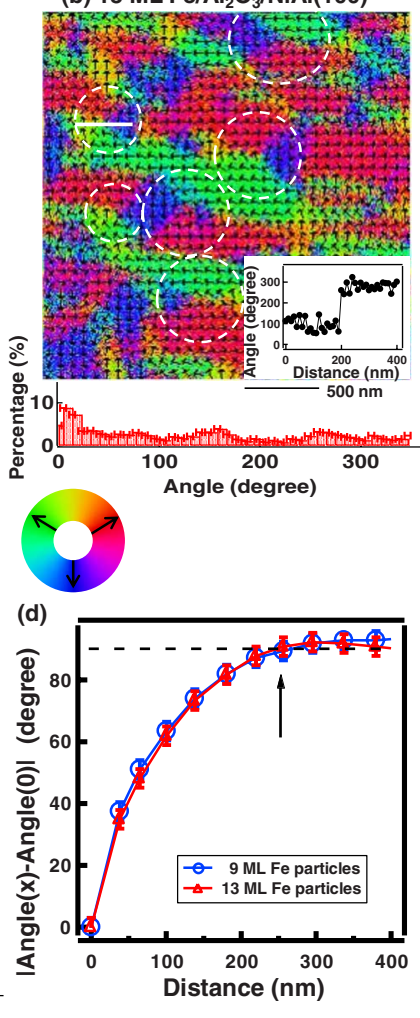

FIG. 3. (Color online) SEMPA images of (a) 9 ML Fe nanoparticles, (b) 13 ML Fe nanoparticles, and (c) 9 ML Fe film with the histogram of the magnetization directions. The arrows indicate the magnetization directions. Angle $=0^{\circ}$ in the histogram is parallel to [010]. The white circles in (b) mark the flux closure magnetic domain structures. The inset exhibits the rotation of magnetization angle along the indicated line crossing the center of a flux closure domain. (d) The correlation functions of 9 and 13 ML Fe nanoparticles, indicating the magnetic correlation length to be $\sim 250 \mathrm{~nm}$.

assemblies, which accordingly costs less energy to create domain walls. Moreover, the circles in Fig. 3(b) point out the flux closure (vortexlike) magnetic domain structures. The density of such kind structures are quite high and the extent ranges a few hundreds $\mathrm{nm}$. The inset shown in Fig. 3(b) reveals a line profile crossing the center of one vortexlike domain. The switch of magnetization happens within one pixel $(\sim 10 \mathrm{~nm})$, indicating that the center area of the vortexlike domain structures is down to $\sim 10 \mathrm{~nm}$, close to the limit of the particle size.

The pixel size in the SEMPA images [Figs. 3(a) and 3(b)] is $10 \mathrm{~nm} \times 10 \mathrm{~nm}^{2}$, very close to the average interparticle distance shown in Fig. 1. In Figs. 4(a) and 4(b), the SEMPA and STM images of the same scale for 13 ML Fe nanoparticles are exhibited, in order to reveal the flux closure domain structure formed by nanoparticles. Up to now, many theoretical simulations ${ }^{17,22}$ and experiments ${ }^{15,17}$ were performed to study the unique magnetic properties of nanoparticle assemblies, especially (1) the reduced magnetic correlation length and (2) flux closure (vortexlike) magnetic domain structures. In the studies of J. F. Löffler et al. ${ }^{23}$ by neutron scattering, the magnetic correlation length of Fe nanoparticle assemblies
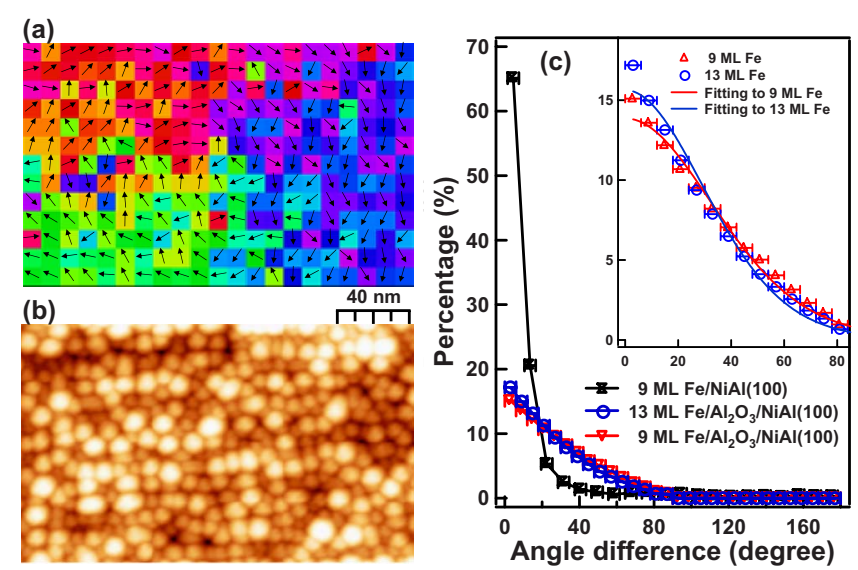

FIG. 4. (Color online) (a) SEMPA and (b) STM images of the same scale for $13 \mathrm{ML}$ Fe nanoparticles are exhibited, in order to reveal the idea of that each pixel represents the magnetization in a $10 \times 10 \mathrm{~nm}^{2}$ area, close to the limit of the particle size. (c) Histogram of angle differences between nearby pixels in SEMPA images of $9 \mathrm{ML}$ Fe nanoparticles, $13 \mathrm{ML}$ nanoparticles, and $9 \mathrm{ML}$ films (as shown in Fig. 3.) The inset shows the magnified histogram of 9 and $13 \mathrm{ML}$ Fe nanoparticles with fitting curves according to Eq. (1).

ranges from 100 to $120 \mathrm{~nm}$ for $10-15 \mathrm{~nm}$ diameter size. The Monte Carlo simulations of A. J. Bennett et al. ${ }^{22}$ and M. Georgescu et al. ${ }^{17}$ show that the magnetic moments of nanoparticles arrange themselves in flux closure structures. In our studies, SEMPA is only little affected by morphology and gives a vector signal that is directly proportional to magnetization. The observation of magnetization direction with high resolution, in Fig. 3, indeed provides conclusive evidences to support the aforementioned simulations and indirect experiments. Further quantitative statistics thus can be carried out for more thorough understanding.

The quantitative statistics is made for detailed discussion of magnetic interaction. Figure 4(c) summarizes the distribution of the angle difference between the nearest-neighbor pixels. For comparison, the histogram of $9 \mathrm{ML} \mathrm{Fe}$ film is also exhibited. The distribution is apparently narrower for the $\mathrm{Fe}$ film, indicating a stronger magnetic coupling. The broad distribution in Fe nanoparticles might be attributed to the weak particle-particle interaction. From the simplified dipolar interaction, one may assume the magnetic interaction energy $E_{I}$ is proportional to $\vec{M}_{1} \cdot \vec{M}_{2} \cdot{ }^{15} M_{1}$ and $M_{2}$ are magnetic moments of two nearest neighbors. Thus $E_{I}$ is written as $E_{I}=$ $-E \cdot \cos (\Delta \theta) . \Delta \theta$ is the nearest-neighbor angle difference. According to Boltzman distribution, $f(\Delta \theta) \propto e^{-E_{I} / k_{B} T}$, the distribution of $\Delta \theta$ should be as the following

$$
f(\Delta \theta)=N(E) \cdot e^{E \cdot \cos (\Delta \theta) / k_{B} T}
$$

Here $N(E)$ is the normalization factor, which actually is the zero-order Bessel function. By fitting the experimental distribution with Eq. (1) and $T=300 \mathrm{~K}$, we obtain the coupling energy $E$, which is the only free parameter in the fitting. The coupling energy $E=79 \pm 2 \mathrm{meV}$ and $98 \pm 3 \mathrm{meV}$ for the 9 and 13 ML Fe nanoparticles, respectively. The fitting curves describe the statistic distribution well, as shown in the inset of Fig. 4(c). The deviation around $\Delta \theta=0$ might be attributed 
to the neglected anisotropy energy or other kinds of interparticle interaction, which prefer to align the nearest neighbor nanoparticles to the same direction. Although the particles are grown on an insulating layer, the connection at particle base can't be exactly excluded in the STM images, and the possible exchange coupling through little connected particle base also might exist.

The above analysis is especially meaningful for nanoparticle assemblies, because the pixels represent the magnetization in a $10 \times 10 \mathrm{~nm}^{2}$ area, close to the limit of interparticle distance, as shown in Fig. 1. Some errors might originate from the fact that each pixel may not be exactly at the position of each nanoparticle. The pixels might be at the border of several particles, and thus measure the average magnetization of two or more particles. This average effect of more particles may obscure the fact of the preferred parallel alignment between the nearest neighbors, and thus suppress the high percentage near $\Delta \theta=0$ in Fig. 4(c). Therefore our analysis should give an underestimated distribution at $\Delta \theta=0$, and in turn the underestimated coupling energy. Considering the case of two Fe nanodiscs with diameter $=10 \mathrm{~nm}$, center-tocenter distance $=10 \mathrm{~nm}$ and height $=2 \mathrm{~nm}$, the dipolar coupling energy is $\sim 94 \mathrm{meV}$, which is very close to our fitting results. ${ }^{11,15,22}$ As well know, for small nanoparticles (diameter $\sim 1 \mathrm{~nm})$, the dipolar coupling is very weak $\left(\ll 300 k_{B} T\right)$. However, for larger nanoparticles (diameter $\sim 10 \mathrm{~nm}$, such as our samples), the dipolar coupling can be even higher than $300 k_{B} T$ and might be the origin for the extended domain structures. $^{21}$ Meanwhile, the possible exchange coupling through the little connected particle base also needs to be considered.

The quantitative analysis shows that the nearest-neighbor dipolar interaction might be the governing factor for the col- lective behavior of $\mathrm{Fe}$ nanoparticle assemblies. With the higher deposition coverage, the particle size is increased, and the interdistance is getting smaller. Thus we have clear increase in the coupling energy $E$, for the higher coverage. From the technical application's point of view, this study points out the direction of controlling the reduced coupling and magnetic anisotropy to form the desired magnetic behaviors, such as magnetic-correlation length, Curie temperature, and domain configuration, by just tuning the particle size, interdistance, or alignment.

\section{SUMMARY}

In this study, in situ SEMPA and STM are employed due to their high resolution in magnetic and topographic imaging. By using SEMPA, we have investigated the detailed microscopic characteristics of the magnetic nanoparticle assemblies such as the high density of flux closure domain structures (size $\sim$ a few hundreds $\mathrm{nm}$ ), and the reduced magnetic correlation length $(\sim 250 \mathrm{~nm})$. The simplified model describes the interparticle coupling distribution well, indicating that the dominant role might be played by the dipolar interaction. These observations will be valuable for fabricating magnetic nanoparticle assemblies with the desired magnetic properties.

\section{ACKNOWLEDGMENTS}

This work was supported by the National Science Council of Taiwan under Grants No. NSC 96-2120-M-002-011, No. NSC 95-2112-M-002-051-MY3, and No. NSC 96-2112-M003-015-MY3. A portion of this research at Oak Ridge National Laboratory's Center for Nanophase Materials Sciences was sponsored by the Scientific User Facilities Division, Office of Basic Energy Sciences, U.S. Department of Energy.

\footnotetext{
*wclin@ntnu.edu.tw

$\dagger$ mtlin@phys.ntu.edu.tw

${ }^{1}$ H.-J. Freund, Surf. Sci. 500, 271 (2002).

${ }^{2}$ M. Bäumer, M. Frank, M. Heemeier, R. Kühnemuth, S. Stempel, and H.-J. Freund, Surf. Sci. 454-456, 957 (2000).

${ }^{3}$ M. Heemeier, S. Stempel, Sh. K. Shaikhutdinov, J. Libuda, M. Bäumer, R. J. Oldman, S. D. Jackson, and H.-J. Freund, Surf. Sci. 523, 103 (2003).

${ }^{4}$ S. G. Grancharov, H. Zeng, S. Sun, Shan X. Wang, S. O'Brien, C. B. Murray, J. R. Kirtley, and G. A. Held, J. Phys. Chem. B 109, 13030 (2005).

${ }^{5}$ J. Xie, S. Peng, N. Brower, N. Pourmand, Shan X. Wang, and S. Sun, Pure Appl. Chem. 78, 1003 (2006).

${ }^{6}$ S. Sun, Hao Zeng, David B. Robinson, S. Raoux, Philip M. Rice, Shan X. Wang, and G. Li, J. Am. Chem. Soc. 126, 273 (2004).

${ }^{7}$ S. A. Majetich and M. Sachan, J. Phys. D 39, R407 (2006).

${ }^{8}$ M. R. Scheinfein, K. E. Schmidt, K. R. Heim, and G. G. Hembree, Phys. Rev. Lett. 76, 1541 (1996).

${ }^{9}$ S. Tomita, K. Akamatsu, H. Shinkai, S. Ikeda, H. Nawafune, C. Mitsumata, T. Kashiwagi and M. Hagiwara, Phys. Rev. B 71 180414(R) (2005).

${ }^{10}$ E. Y. Vedmedenko, N. Mikuszeit, H. P. Oepen, and R. Wiesen-
}

danger, Phys. Rev. Lett. 95, 207202 (2005).

${ }^{11}$ P. Poddar, T. Telem-Shafir, T. Fried, and G. Markovich, Phys. Rev. B 66 060403(R) (2002).

${ }^{12}$ O. Petracic, A. Glatz, and W. Kleemann, Phys. Rev. B 70, 214432 (2004).

${ }^{13}$ U. Schlickum, W. Wulfhekel, and J. Kirschner, Appl. Phys. Lett. 832016 (2003); A. Kubetzka, O. Pietzsch, M. Bode, and R. Wiesendanger, Phys. Rev. B 63 140407(R) (2001).

${ }^{14}$ V. F. Puntes, P. Gorostiza, D. M. Aruguete, N. G. Bastus, and A. P. Alivisatos, Nat. Mater. 3, 263 (2004).

${ }^{15}$ M. Georgescu, J. L. Viota, M. Klokkenburg, B. H. Erné, D. Vanmaekelbergh, and P. A. Zeijlmans van Emmichoven, Phys. Rev. B 77, 024423 (2008).

${ }^{16}$ S. A. Koch, R. H. te Velde, G. Palasantzas, and J. Th. M. De Hosson, Appl. Phys. Lett. 84, 556 (2004).

${ }^{17}$ M. Georgescu, M. Klokkenburg, B. H. Erné, P. Liljeroth, D. Vanmaekelbergh, and P. A. Zeijlmans van Emmichoven, Phys. Rev. B 73, 184415 (2006).

${ }^{18}$ H. P. Oepen and J. Kirschner, Scanning Microsc. 5, 1 (1991).

${ }^{19}$ W. C. Lin, C. C. Kuo, M. F. Luo, K.-J. Song, and M.-T. Lin, Appl. Phys. Lett. 86, 043105 (2005). 
${ }^{20}$ W. C. Lin, S. S. Wong, P. C. Huang, C. B. Wu, B. R. Xu, C. T. Chiang, H. Y. Yen, and M.-T. Lin, Appl. Phys. Lett. 89, 153111 (2006).

${ }^{21}$ W. C. Lin, P. C. Huang, K.-J. Song, and M.-T. Lin, Appl. Phys.
Lett. 88, 153117 (2006).

${ }^{22}$ A. J. Bennett and J. M. Xu, Appl. Phys. Lett. 82, 2503 (2003).

${ }^{23}$ J. F. Löffler, H.-B. Braun, and W. Wagner, Phys. Rev. Lett. 85, 1990 (2000). 